\title{
14. Über die Grössenordnung des absoluten Betrages von einer linearen inhomogenen Form, VIII. $\left(^{(1)}\right.$ Über einen Satz von Khintchine.
}

\author{
Von Seigo MoRImoto, Hiroshima.
}

(Eingegangen am 25, Januar, 1938.)

1. Herr Khintchine hat den folgenden Satz bewiesen $\left({ }^{2}\right)$.

Khintchinescher Satz. Wenn a eine irrationale Zahl ist und die Gleichung $\alpha x-y+\beta=0$ keine ganzzählige Lösung $(x, y)$, wo $x>0$, hat, wird die Ungleichung

$$
|\alpha x-y+\beta|<\frac{1+\varepsilon}{\sqrt{5} x}
$$

durch unendlich viele Paare ganzer Zahlen $x, y$, wo $x>0$, befriedigt.

Dieser Satz suggeriert uns eine neuere Richtung unsres Problems, $d . h$. die Untersuchung der Grössenordnung von $|\alpha x-y+\beta|$ in einer Halbebene. Wie er bemerkt, kann der Satz in dieselben Formen nicht mehr verschärft werden, weil die Gleichung $\alpha x-y+\beta=0$ in der Halbebene $x \leqq 0$ ganzzählige Lösung haben kann. Aber wir können vermuten, dass in dem Falle, wo die Ungleichung (1) nicht mehr verschärft werden kann, die Gleichung $x x-y+\beta=0$ in $x \leqq 0$ eine Lösung oder eine sehr scharf angenäherte Lösung hat. Diese Ủberlegung führt mich nach der Aufgabe, die Beziehung $z$ wischen die unteren Grenzen von $|x(\alpha x-y+\beta)|$ für die beiden Halbebenen $x>0$ und $x \leqq 0$ zu untersuchen.

2. In dem ersten Teile dieser Arbeit $\left({ }^{3}\right)$ habe ich die Näherungspunkte von der Form $\alpha x-y+\beta$ definiert und die Beziehung zwischen diesen Punkten untersucht. Insbesondere habe ich gezeigt, dass es unendlichviele Gruppen der Näherungspunkte $A^{\prime}, B^{\prime}, C^{\prime}, D^{\prime}$ gibt, von der Beschaffenheit, dass sie die Ecken einer Parallelogramm von dem Inhalt 1 bilden und von denen $A^{\prime}$ und $B^{\prime}, C^{\prime}$ bzw. $D^{\prime}$ in I, II bzw. IV liegen, wo I, II, III, IV die vier licht.

(1) Ein Teil dieser Arbeit habe ich in Osaka Sijo Danwakaisi No. 98 (1936) veröffent.-

(2) Neuer Beweis und Verallgemeinerung eines Hurwitzschen Satzes, Math. Ann. 111, $631-637$.

(3) Teil I dieser Arbeit ist in Vol. 3 (1926), 1-26 dieses Journals veröffentlicht. Teil II ebenda 91-106. Teil III, 4 (1927), 41-48. Teil IV, ebenda, 149-167. Teil V, 5 (1929), 295-316. Teil VI, 6 (1929), 349-362. Teil VII (Number der Teilen wegnehmend) Tohoku Math. Journal, 38 (1933), 7-33. I-IV unter meinem früheren Familliennahmen S, Fukasawa。 
Teilen der Ebene bedeuten, in welchen die Ebene von $L: \alpha x-y+\beta=0$ und $Y$-Achse geteilt wird. (Die vier Näherungspunkte $P_{m}, P_{l}^{\prime \prime}, P_{n}, Q_{n}$ in den Seiten 6-7 dort. Diese Tatsache können wir leicht beweisen.) Wir führen durch die Affintransformation, die den Inhalt und $Y$-Ache nicht ändert, die Gerade $L$ in $X$-Achse, die Teilen I, II, III, bzw. IV in ersten, zweiten, dritten bzw. vierten Quadranten und $C^{\prime}$ in einem Punkte mit den Koordinaten $(-a, a)$. Seien $B, D, A$ die neuen Lagen der Punkte $B^{\prime}, D^{\prime}, A^{\prime}$ und $(x, y),(u,-v),(x+u+a, y-v-a)$ ihre Koordinaten. Bezeichnet man z. B. mit $\varphi\left(A^{\prime}\right)$ die Werte von $\mid x(\alpha x-y+\beta \mid$ für die Koordinaten $(x, y)$ von $A^{\prime}$, so ist

$$
\phi\left(B^{\prime}\right)=x y, \quad \phi\left(C^{\prime}\right)=\alpha^{2}, \quad \phi\left(D^{\prime}\right)=u v, \quad \phi\left(A^{\prime}\right)=(x+u+a)(y-v-a) .
$$

Wir halten a fest und suchen die obere Grenze von

$$
k=\operatorname{Min}(x y, u v,(x+u+\alpha)(y-v-a)) .
$$

3. Zuerst zeigen wir, dass diese obere Grenze nur in dem Falle

$$
x y=u v=(x+u+a)(y-v-a)
$$

erreicht werden kann. $\mathrm{Zu}$ diesem $\mathrm{Zwe}$ we betrachten wir die Hyperbeln

$$
x y= \pm k \text {. }
$$

Die Punkte $A, B, D$ liegen alle innenhalb oder auf der Grenze von (3) und wenigstens eine von ihnen liegt auf der Grenze. Wir verschieben die Strecke $A B$ auf sich selbst, bis ihr Mittelpunkt mit dem Mittelpunkte der Sehne, die die Gerade $A B$ von (3) schneidet, übereinstimmt und $k$ so gross bestimmen, dass $A$ und $B$ oder $D$ auf (3) liegn. Diese Lagen der Punkte $A, B, D$ und den Inhalt des Parallelogramms $A B C D$ festhaltend, durch Vergrössern von $k$, können wir erreichen, dass alle Punkte $A, B, D$ auf (3) liegen. Darum braucht man nur den Fall (2) zu betrachten.

4. Weil der Inhalt von $A B C D$ gleich 1 ist,

$$
(x+a)(v+a)+(y-a)(u+a)=1 \text {. }
$$

Wir setzen

$$
x y=z
$$

und eliminieren $x, y, v$ aus (2), (4) und (5). Wir haben aus (4)

$$
\left.\begin{array}{c}
x v+a v+a x+y u-a u+a y=1 \\
-x v-a v-a x+y u-a u+a y-a^{2}-u v=0
\end{array}\right\}
$$




$$
\begin{aligned}
& 2 y u-2 a u+2 a y-a^{2}-u v=1 \\
& \left.2 x v+2 a v+2 a x+a^{2}+u v=1\right\} \\
& 2 \frac{z}{x} u-2 a u+2 a \frac{z}{x}-a^{2}-z=1 \\
& 2 x \frac{z}{u}+2 a \frac{z}{u}+2 a x+a^{2}+z=1 \\
& \frac{z}{x}=\frac{1+2 a u+a^{2}+z}{2(a+u)} \\
& \left.x=\frac{1-2 a \frac{z}{u}-a^{2}-z}{2\left(\frac{z}{u}+a\right)}\right\} \\
& z=\left(1+2 a u+a^{2}+z\right)\left(1-2 a \frac{z}{u}-a^{2}-z\right) \\
& 4\left(\frac{z}{u}+a\right)(a+u)
\end{aligned}
$$$$
3 a\left(3 z+a^{2}-1\right) u^{2}+\left(5 z^{2}+10 a^{2} z+z^{4}-1\right) u+2 a z\left(3 z+a^{2}+1\right)=0 .
$$

Diese Gleichung nuss eine positive Wurzel haben. Darum besteht eine der beiden Ungleichungen

$$
\begin{aligned}
& 3 z+a^{2}-1<0 \\
& 5 z^{2}+10 \alpha^{2} z+a^{4}-1<0,
\end{aligned}
$$

weil das konstante Glied der Gleichung positive ist. Aus (7) und (8)

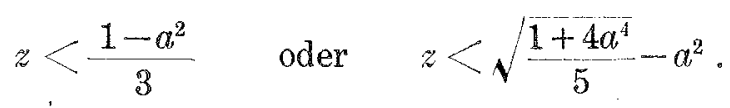

4. Wenn wir $\frac{1-a^{2}}{3}<\sqrt{\frac{1+4 a^{4}}{5}}-a^{2}$ setzen, so haben wir

$$
\left(1-a^{2}\right)\left(1-4 a^{2}\right)>0 \text {. }
$$

Da $a<1$ ist, $\quad \frac{1-a^{2}}{3} \leqq \sqrt{\frac{1+4 a^{4}}{5}-a^{2} \quad \text { für } \quad a^{2} \leqq 1}$

$$
\frac{1-a^{2}}{3}>\sqrt{\frac{1+4 a^{4}}{5}-a^{2}} \text { für } \quad a^{2}>\frac{1}{4},
$$

also

$$
\approx<f\left(a^{2}\right)
$$


Wo

$$
\begin{aligned}
f\left(a^{2}\right) & =\frac{1-a^{2}}{3} & & \text { für } \quad a^{2}>\frac{1}{4} \\
& =\sqrt{\frac{1+4 \alpha^{2}}{5}-a^{2}} & & \text { für } \quad a^{2} \lesseqgtr \frac{1}{4} .
\end{aligned}
$$

Wir bemerken dabei, dass $f^{\prime}\left(a^{2}\right)$ eine abnehmende Funktion von $a^{2}$ ist. Bezeichnet man

$$
\lim _{x>0}|x(\alpha x-y+\beta)|=P, \quad \lim _{x<v}|x(\alpha x-y+\beta)|=N \quad \text { für die ganzen } x, y,
$$

so ist $N \leqq a^{2}, P \leqq$ Min. $(x y, u v,(x+u+a)(y-v-a))$. Daher haben wir

$$
P \leqq f\left(\alpha^{2}\right) \leqq f(N)=\sqrt{\frac{1+4 N^{2}}{5}}-N \quad \text { für } \quad N \leqq 1 / 4
$$

Wenn $N>1 / 4$ ist, so ist $P \leqq f\left(a^{2}\right) \leqq f(N)=\frac{1-N^{2}}{3}<1 / 4$. Wir denken dabei die rechte und die linke Seite von $Y$-Achse vertauscht, so haben wir

$$
\begin{aligned}
& N \leqq \sqrt{\frac{1+4 P^{2}}{5}}-P \\
& P^{2}+10 N P+5 N^{2}-1 \leqq 0 \\
& P \leqq \sqrt{1+20 N^{2}}-5 N .
\end{aligned}
$$

Dies gibt ein schärferes Resultat als die Ungleichung $P<(1-N) / 3$, weil, wenn wir

$$
\frac{1-N}{3} \geq \sqrt{1+20 N^{2}}-5 N
$$

setzen, so die evidente Ungleichung $(4 N-1)(N+2) \geqq 0$ folgt. Daraus haben wir den

Satz I.

$$
\left.\begin{array}{ll}
P \leqq \sqrt{\frac{1+4 N^{2}}{5}-N} & \text { für } \quad N \leqq 1 / 4 \\
P \leqq \sqrt{1+20 N^{2}}-5 N & \text { für } \quad N>1 / 4
\end{array}\right\}
$$

Bemerkung. Da die rechte Seite von (11) nicht grösser als $f(N) \leqq 1 / \sqrt{5}$ ist, enthält dieser Satz den KHINTCHINEschen Satz als einen speziellen Fall. Da aber die rechte Seite von (11) im Falle $N \geqq 1 / 4$ kleiner als $1 / 4$ ist, d. h. $\operatorname{Min}(P, N) \leqq 1 / 4$, enthält dieser Satz auch den Minkowskischen Satz.

5. Zunächst denken wir die Bedingung dafür, dass die Gleichung (6) reelle Wurzel hat, nämlich

$$
\left(5 z^{2}+10 a^{2} z+a^{4}-1\right)^{2} \geq 4 a^{2} z\left\{\left(3 z+a^{2}\right)^{2}-1\right\} .
$$


Unter den Bedingungen

$$
\begin{array}{ll} 
& a^{2} \leqq 1 / 4, \quad z<f\left(a^{2}\right) \\
\text { ist } \quad & 5 z^{2}+10 \alpha^{2} z+a^{4}-1<0 .
\end{array}
$$

Daher nimmt die linke Seite von (12) von $\left(a^{4}-1\right)^{2}$ bis 0 monoton ab, wenn $z$ von 0 bis $f\left(a^{2}\right)$ zunimmt. Die rechte Seite ist aber negativ für $z<\frac{1-a^{2}}{3}$, null für $z=\frac{1-a^{2}}{3}$ und nimmt monoton mit $z$ zu für $z>\frac{1-a^{2}}{3}$. Daher hat

$$
\left(5 z^{2}+10 a^{2} z+a^{4}-1\right)^{2}-4 a^{2} z\left\{\left(3 z+a^{2}\right)^{2}-1\right\}=0
$$

eine und nur eine Wurzel $z_{1}$ zwischen $\left(1-a^{2}\right) / 3$ und $f\left(\alpha^{2}\right)$ und besteht (12) für $z \leqq z_{1}$. Überdies nimmt die linke Seite von (12) ab und die rechte Seite zu, wenn $z$ konstant bleibt und $a^{2}$ zunimmt. Daher ist

$$
z_{1}=F\left(a^{2}\right)
$$

eine monoton abnehmende Funktion von $a^{2}$ für $a^{2} \leqq 1 / 4$. Folglich hat $F\left(a^{2}\right)$ auch die monoton abnehmende Umkehrfunktion $a^{2}=F^{-1}\left(z_{1}\right)$ für $0 \leqq z_{1} \leqq 1 / 4$ $\left(\frac{1}{4}=F\left(\frac{1}{4}\right)\right)$. Daraus haben wir durch die ähnliche Überlegung wie oben den folgenden

Satz II. $P \leqq F(N)$ für $N \leqq 1 / 4$ und

$$
P \leqq F^{-1}(N) \quad \text { fiur } N>1 / 4 \text {. }
$$

6. Endlich suchen wir nach der angenäherte Darstellung von dem Satz II. Sei $g\left(a^{2}\right)$ eine Funktion von $a$, welche die folgenden Ungleichungen befriedigt,

$$
\begin{gathered}
\frac{1-a^{2}}{3} \leqq g(a) \leqq \sqrt{\frac{1+4 a^{4}}{5}-a^{2}} \\
G\left\{g\left(a^{2}\right)\right\}<0
\end{gathered}
$$

wo $G(z)$ die linke Seite von (12) bedeutet, so ist

$$
F\left(\alpha^{2}\right)<g\left(a^{2}\right)
$$

und haben wir das Resultat

$$
z<g\left(\alpha^{2}\right) \quad \text { oder } \quad P<g(N) \text {. }
$$

Fưr $a^{2} \leqq 1 / 4$ haben wir die Entwicklung 


$$
z=\frac{1}{5}-\frac{2 a}{5 \sqrt[4]{ }}-\cdots \cdot
$$

Nun setze ich

$$
g\left(a^{2}\right)=g_{1}\left(a^{2}\right)=\frac{1}{\gamma^{\prime}}-\frac{2 \alpha}{5 \sqrt[4]{5}}-\frac{9 a^{2}}{25}
$$

so folgt

$$
\begin{aligned}
G\left\{g_{1}\left(a^{2}\right)\right\}=16( & -\frac{7 a^{3}}{25 \sqrt{5}}+\frac{98 a^{4}}{125}-\frac{241 a^{5}}{125 \sqrt{5} \sqrt{5}}-\frac{777 a^{6}}{625 \sqrt{5}} \\
& \left.+\frac{2008 a^{7}}{3125 \sqrt[4]{5}}+\frac{3730 a^{8}}{15625}\right)
\end{aligned}
$$

Bezeichnet man mit

$$
\begin{gathered}
\quad \frac{a}{\sqrt{5}-5}=t \\
G\left(g_{1}\left(a^{2}\right)\right)=16 t^{3}\left(-7+98 t-241 t^{2}-777 t^{3}+2008 t^{4}+3730 t^{5}\right) \\
=16 t^{3} \phi(t),
\end{gathered}
$$

so ist $\frac{d}{d t}\left(-7+98 t-241 t^{2}-777 t^{3}\right)=98-482 t-2331 t^{2}>0$ für $0<t<0.12$. Folglich ist $\varphi(t)$ eine zunehmende Funktion in $0<t<0.12$. Weil $\varphi(t)=0$ eine Wurzel $t=0.1042$ hat, haben wir

$$
\begin{array}{rll}
\varphi(t)<0 \quad \text { fuir } & t \leqq 0.1042 \\
& \text { also } & a \leqq 0.3484 \\
& & a \leqq 0.1214
\end{array}
$$

d. h.

$$
\begin{aligned}
& G\left(g_{1}\left(a^{2}\right)\right)<0 \\
& f\left(a^{2}\right)<g_{1}\left(a^{2}\right) \quad \text { für } \quad a \leqq 0.1214 .
\end{aligned}
$$

7. Zunächst betrachten wir die Umgebung von $a^{2}=1 / 4$. Zu dieser Zwecke setzen wir

$$
t=z-\frac{1}{4}, \quad b=\frac{1}{4}-a^{2}
$$

Dann geht $G(z)$ über in

$$
\begin{aligned}
G(z)=G_{1}(t)= & \left(5 t^{2}+5 t-10 b t-3 b+b^{2}\right)^{2} \\
& -(3 t-b)(3 t-b+2)(1-2 b)\left(t+\frac{1}{4}\right) .
\end{aligned}
$$




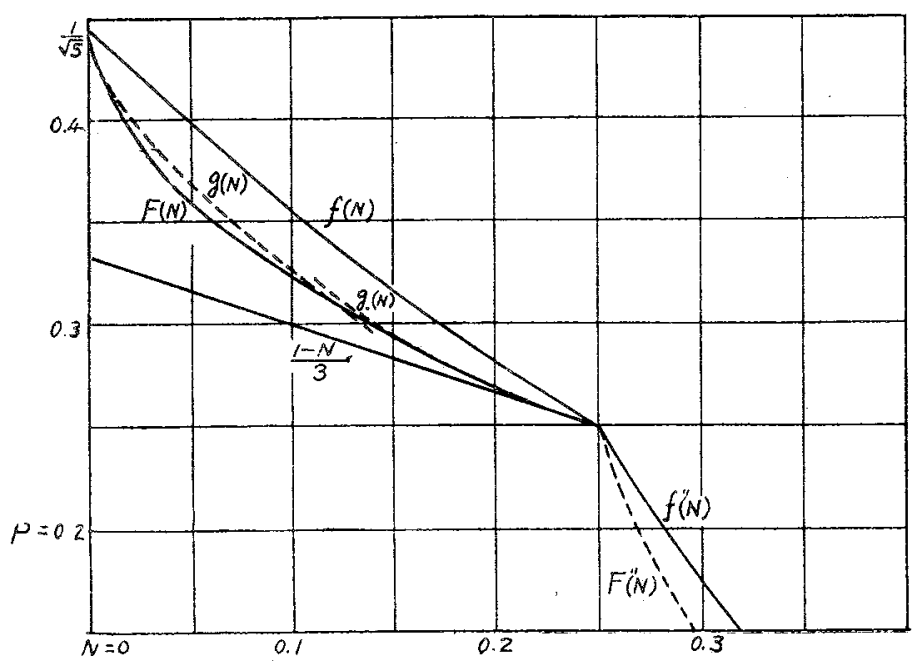

Jch setze

$$
g\left(a^{2}\right)=g_{2}\left(a^{2}\right)=\frac{1}{4}+\frac{1}{3}\left(\frac{1}{4}-a^{2}\right)+\frac{11}{9}\left(\frac{1}{4}-a^{2}\right)^{2},
$$

also

$$
t=\frac{b}{3}+\frac{11 b^{2}}{9}
$$

so folgt

$$
\begin{aligned}
G_{1}(t)= & \left(\frac{4}{3} b-\frac{13}{3} b^{2}+\frac{220}{27} b^{3}-\frac{605}{81} b^{4}\right)^{2} \\
& +\frac{11}{3} b^{2}\left(\frac{11}{3}+b^{2}+2\right)(4 b-1)\left(\frac{1}{4}-\frac{b}{3}+\frac{11 b^{2}}{9}\right) \\
< & \left(\frac{4}{3} b-\frac{13}{3} b^{2}+\frac{74}{9} b^{3}-\frac{67}{9} b^{4}\right)^{2}+\frac{11 b^{2}}{9^{2} \times 4}\left(6+11 b^{2}\right)(4 b-1)\left(9+12 b+44 b^{2}\right) \\
= & \frac{a_{1}^{\prime}}{9^{?} \times 4}\left(-18-2160 b+12363 b^{2}-15000 b^{3}\right. \\
& \left.\quad+43292 b^{4}-18368 b^{5}+17956 b^{6}\right) .
\end{aligned}
$$

Für $b<0.15$

$$
\begin{aligned}
& -18-2160 b+12363 b^{2}-15000 b^{3}+43292 b^{4}-18368 b^{5}+17956 b^{6} \\
& <-18-2160 b+1855 b-15000 b^{3}+6494 b^{3}-18368 b^{5}+2694 b^{5} \\
& <0
\end{aligned}
$$

d. h. für $b<0.15$, also $0.1<a \leqq 1 / 4$ 


$$
\begin{aligned}
& G\left(g_{2}\left(a^{2}\right)\right)<0 \\
& f\left(a^{2}\right)<g\left(a^{2}\right) .
\end{aligned}
$$

8. Wie vorher haben wir (15) und (16). Da

$$
\begin{aligned}
\text { für } & a^{2}=0.1214 & & g\left(a^{2}\right)=f\left(a^{2}\right)=0.3103 \\
\text { und } & =0.1257 & & g\left(a^{2}\right)=g(0.1214)=0.3103
\end{aligned}
$$

ist, können wir $g\left(a^{2}\right)=0.3103$ für $0.1214<a^{2}<0.1257$ setzen. Ủberdies bemerkt man, dass

$$
\begin{aligned}
& g_{1}^{-1}(z)=\frac{5 \sqrt{5} \sqrt{10-9 \sqrt{5} z-1}}{81} \\
& g_{2}^{-1}(z)=\frac{17-6 \sqrt{44 z-10}}{44} .
\end{aligned}
$$

Daraus haben wir den

Satz III.

$$
P<H(N)
$$

wo

$$
\begin{aligned}
& H(N)=\frac{1}{5}-\frac{2 \sqrt{N}}{5 \sqrt[4]{5}}-\frac{9 N}{25} \\
& H(N)=0.3103 \\
& H(N)=\frac{1-N}{3}+\frac{11}{9}\left(\frac{1}{4}-N\right)^{2} \quad \text { für } \quad 0.1257<N \leqq 0.25 \\
& H(N)=\frac{17-6 \sqrt{4} 4 N-10}{44} \\
& H(N)=\frac{5 \sqrt{5} \sqrt{10-9 \sqrt{5} N}-1}{81} \text { fü } 0.3103<N \leqq 0.4472 \\
& \text { für } 0 \leqq N \leqq 0.1214 \\
& \text { für } 0.1214<N \leqq 0.1257 \\
& \text { für } 0.25<N<0.3103 \\
& =1 / \sqrt{5} \text {. }
\end{aligned}
$$

